\title{
Mobile Trailer Backup Assist System
}

\author{
Kruglov S.P. \\ FGBOU VO "Irkutsk State University of Railway \\ Engineering" (ISTU) \\ Irkutsk, Russia \\ e-mail: kruglov_s_p@mail.ru
}

\author{
Kovyrshin S.V. \\ FGBOU VO "Irkutsk State University of Railway \\ Engineering" (ISTU) \\ Irkutsk, Russia \\ e-mail: sergkow@mail.ru
}

\begin{abstract}
We propose an approach for creation of a mobile version of a Trailer Backup Assist System. The System consists of a mobile device (tablet, smartphone, etc.) with a specialized software and two easily removable sensors with a wireless communication channel to determine the steering angle of the driver and the hitch angle. As the adjustable parameter, we use the hitch angle set by the driver according to the maneuvering conditions. The main tasks of the mobile device are: preliminary estimation of the System parameters based on the identification algorithm, predetermined hitch angle input, and generation of voice and graphic commands for the driver's steering wheel rotation in the desired direction. In comparison with the known similar systems, this System is easy to mount and does not require the use of the car regular systems; it is cheap and has low operational costs. A model example is given.
\end{abstract}

Keywords - trailer; backup; driver assistance; mobile system, estimation, identification algorithm.

\section{INTRODUCTION}

Trailer backup parking may be problematic for both young and experienced drivers. Drivers who own trailers were questioned, and the poll showed that more than $70 \%$ of them can't do trailer backup parking. The special feature of this movement consists of the fact that moving backwards is necessary to compensate for small deviations of the trailer from a straight movement by steering in the opposite direction. Inaccurate control by the driver can lead to the jackknifing of the system (high value of the hitch angle); one can change this position by moving forward only.

To help the driver during the trailer backup movement, the Driver Assist System should take into account the intention of the driver, analyze the current value of the hitch angle, steering wheel angle of the driver, and generate hints to the driver of the vehicle in order the trailer to move steadily, in a given direction, with the car-trailer jackknifing eliminated.

Well-known works on this subject with creation of systems based on conventional PI-controllers, neurocontrollers, fuzzy logic, obtaining of optimal solutions, such as [1-6], etc. The Trailer Backup Assist Systems are already actively introduced by the leading vehicle producers, numerous patents are received, for example, [7-9], they implement the tracking of the trailer track curvature set by the driver.
But the main disadvantage of the existing systems is that they are mounted at the car manufacturing plants or in a specialized service center with introduction to the vehicle control regular systems, and are configured for one car only. They are not cheap. That is why there is no possibility of lowcost mobile installation of such assist systems to the medium cars. There is also no possibility of reinstalling from one car to another. For assist systems used, the presence of regular video system and other special equipment, etc. is mandatory.

In addition, many well-known driver assist systems require pre-configuration of control system parameters with preliminary determination of those. There are solutions for automatic identification of one of these parameters - the length of the trailer, using ultrasonic parking sensors, with the use of radio transmitting devices, with test movement of the system in steady rotation [10-13], etc. However, they do not define all the parameters as necessary for the control law synthesis.

The objective of this study is to develop such a Trailer Backup Assist System that does not require intervention to regular systems of a car, is not expensive, easy to install, mobile for re-installation to another vehicle and has low operational costs for system setup.

The objective can be achieved by means of implementation of it based on a mobile computing device, such as smartphone or tablet, with specialized software, as well as the easy-removable steering wheel angle sensor and the trailer hitch angle sensor with wireless info transmission. The task of the mobile device is to implement the two major modes: auto-setup and operating mode. When auto-setup mode by the data from sensors during the test forward movement based on the identification algorithm is on, there occurs definition of parameters necessary for the control law synthesis, and storage of them. In the operating mode, the driver sets up the desired hitch angle, and then he creates commands to the driver (voice and/or graphic) for the desired driver wheel deflection on the basis of sensor data. The driver performs these commands. The following are the construction features of such a system.

\section{TRAILER SYSTEM MATHEMATICAL MODEL}

In Fig. 1 there is a kinematic diagram of a controlled trailer. 


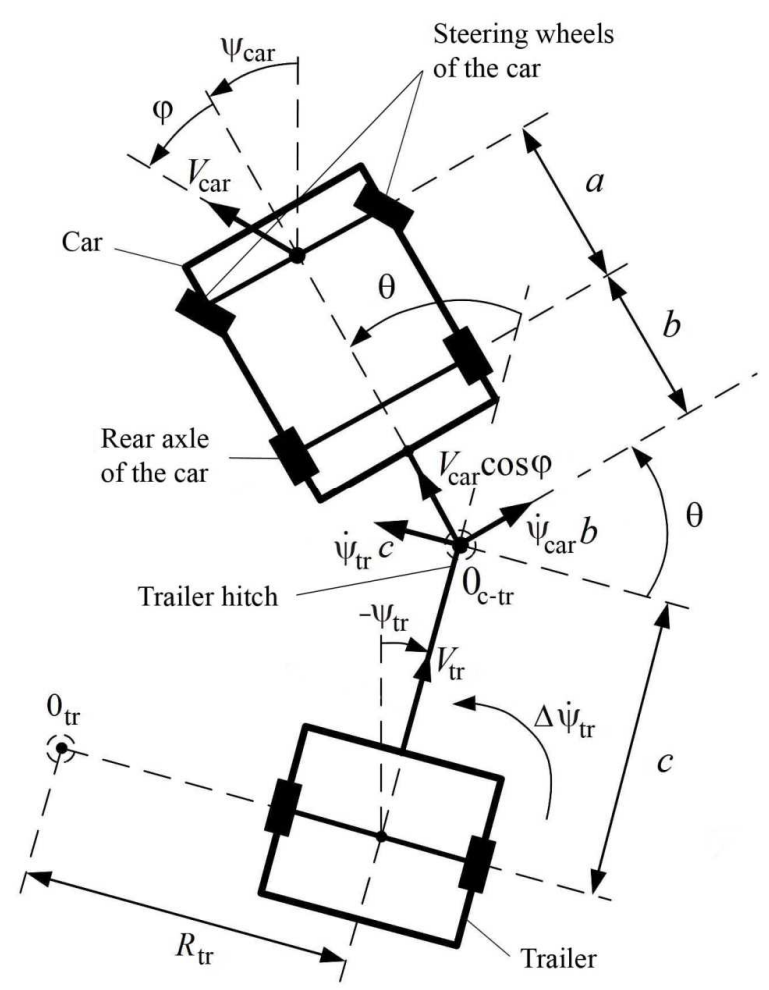

Fig.1 Kinematic diagram of a controlled trailer

In Fig. 1 and in the subsequent discussion the following definitions are used:

$\varphi$ - steering wheels deflection angle of a vehicle with the following typical constraint: $|\varphi| \leq \varphi_{\max } \leq 30^{\circ}$; value $\varphi_{\max }$ will be determined from the following stability condition for forward movement; we assume that deflection of the driver wheel and the deviation of the steering wheels of the vehicle are connected according to the following relation:

$$
\varphi=k_{\mathrm{st}} \varphi_{\mathrm{st}}
$$

where $\varphi_{\text {st }}-$ driver wheel deviation angle, $\mathrm{k}_{\mathrm{st}}-\mathrm{a}$ positive proportion factor for steering wheels control, usually $\mathrm{k}_{\mathrm{st}} \ll 1$; note that the deviation of the driver wheel is limited too: $\varphi_{\text {st } \max }=\varphi_{\max } / k_{\text {st }}$

$\theta$ is the hitch angle or deviation angle of the longitudinal axis of the trailer from the vehicle's longitudinal axis, we assume that $|\theta| \leq \theta_{\max }<90^{\circ}$, where $\theta_{\max }$ is the maximum value of the hitch angle modulus used by the control system; the value of the hitch angle modulus upon exceeding of which during the backward movement it starts involuntary increase with no possibility of compensation due to the limitations of $\varphi$ is called the jackknifing angle $\left(\theta_{\text {jack }}\right)$;

$\psi_{\text {car }}, \psi_{\text {tr }}$ is the vehicle and trailer rotation angle (respectively) from any direction, for example, from specified direction (indicated by a vertical line in the figure);
$V_{\text {car }}, V_{\mathrm{tr}}$ - the speed of the vehicle and trailer, respectively, the vehicle speed will be measured by steering wheels with the attachment point in the center of the steering wheels rotation axis;

$0_{\mathrm{tr}}-$ the center of trailer rotation during a turn;

$0_{c-t r}-$ the trailer rotation point relatively to the vehicle, the hitch point;

$R_{\mathrm{tr}}$ - the trailer rotation radius measured along the trailer wheels rotation axis (effective axis for multi-axis trailer);

$a, b, c$ - kinematic parameters of the controlled trailer ( $a, c$ - positive non-zero numbers), respectively: is the car wheelbase, or the distance between the car steering wheels rotation axis and the rear axle axis; hitch offset, or the distance from the rear axle axis to the hitch point; the length of the trailer, or the distance from the hitch point to the trailer wheels rotation axis (effective axis); note that usually $a>|b|$, $c>|b|$; we assume that if $b<0$, then $c \gg|b|$; an additional requirement to the $c$ parameter shall be defined below;

$\Delta \dot{\psi}_{\mathrm{tr}}$ - the angular trailer velocity caused by external uncontrollable disturbances, for example, the presence of significant road irregularities, the variable soil density occurring on arable land, on snow, on sand, etc.; we assume this value as limited.

For compilation of mathematical description for the controlled trailer, the following assumptions were assumed:

- the car is moving at a relatively low speed;

- tire lateral compliance effect, side skid of the vehicle will not be taken into account;

- movement dynamics - will not be taken into account, in particular, the resulting relations will not depend on accelerations.

It is known that the angular velocity of the vehicle is determined by the following relationship:

$$
\dot{\psi}_{\mathrm{car}}=\frac{V_{\mathrm{car}}}{a} \sin \varphi .
$$

From Fig.1, we can determine that:

$$
\psi_{\mathrm{tr}}=\psi_{\mathrm{car}}-\theta
$$

When the vehicle is in motion, there are two vectors of linear velocity that affect the hitch point: vehicle forward movement vector corresponding to the longitudinal speed of the vehicle $\left(V_{\text {car }} \cos \varphi\right)$, and the $\left(\dot{\psi}_{\text {car }} b\right)$ vector corresponding to the rotational movement of the vehicle. Based on these vectors, we shall determine trailer linear and angular velocity. Using the projections of these vectors on the trailer longitudinal axis, we shall find the speed of the trailer:

$$
V_{\mathrm{tr}}=V_{\mathrm{car}} \cos \varphi \cos \theta+\dot{\psi}_{\mathrm{car}} b \sin \theta
$$


By inserting the formula for $\dot{\psi}_{\text {car }}$ according to the relationship (2), we shall find:

$$
V_{\mathrm{tr}}=V_{\mathrm{car}}\left(\cos \varphi \cos \theta+\frac{b}{a} \sin \varphi \sin \theta\right) \text {. }
$$

Angular velocity of the trailer shall be determined by the linear velocity vector $\left(\dot{\psi}_{\operatorname{tr}} c\right)$, perpendicular to the trailer longitudinal axis (see Fig.1). According to the kinematic scheme, it is clear that when having $\Delta \dot{\psi}_{\mathrm{tr}}=0$ :

$$
\dot{\psi}_{\mathrm{tr}} c=V_{\mathrm{car}} \cos \varphi \sin \theta-\dot{\psi}_{\mathrm{car}} b \cos \theta .
$$

Also, making the insertion in (2), and taking into account the non-zero and disturbing affect, we shall find:

$$
\dot{\psi}_{\mathrm{tr}}=\frac{V_{\mathrm{car}}}{c}\left(\cos \varphi \sin \theta-\frac{b}{a} \sin \varphi \cos \theta\right)+\Delta \dot{\psi}_{\mathrm{tr}}
$$

Since (3) implies that $\dot{\theta}=\dot{\psi}_{\mathrm{car}}-\dot{\psi}_{\mathrm{tr}}$, according to the relationships (2) and (5), it is easy to determine the angle change rate $\theta$ :

$\dot{\theta}=\frac{V_{\mathrm{car}}}{a c}(c \sin \varphi-a \cos \varphi \sin \theta+b \sin \varphi \cos \theta)-\Delta \dot{\psi}_{\mathrm{tr}}$.

When we use the addition formula of trigonometric functions $\alpha \sin \gamma+\beta \cos \gamma=\sqrt{\alpha^{2}+\beta^{2}} \sin (\gamma+\operatorname{arctg}(\beta / \alpha))$ and combine sines and cosines according to $\varphi$, (6) can be represented as:

$$
\dot{\theta}=-\operatorname{sign}\left(V_{\text {car }}\right) k(\theta) \cdot \sin \left[\varphi_{\text {bal }}(\theta)-\varphi\right]-\Delta \dot{\psi}_{\text {tr }}
$$

where $k(\theta) \triangleq(a c)^{-1}\left|V_{\text {car }}\right| \sqrt{(c+b \cos \theta)^{2}+(a \sin \theta)^{2}}$ - is a positive gain (the statement about positivity is easy to deterine out of the accepted conditions);

$$
\varphi_{\mathrm{bal}}(\theta) \triangleq \tan ^{-1}[\mathrm{a} \sin \theta /(\mathrm{c}+\mathrm{b} \cos \theta)] \approx \lambda_{0} \sin \theta
$$

- the balancing dependence of the steering wheels angle according to the corner $\theta$; or such value $\varphi$ that if it is fixed, with $\Delta \dot{\psi}_{\text {tr }} \equiv 0$ given, the current value of the hitch angle will be in the balancing position: $\theta \triangleq \theta_{\text {bal }} \equiv 0$, or controlled trailer will be in a steady rotation, both when moving forward and backwards;

$\lambda_{0} \triangleq \lambda(\theta=0)=a /(b+c)$ - is the parameter $\varphi_{\text {bal }}(\theta)$ function linearization near the point $\theta=0$;

$\lambda(\theta) \triangleq \mathrm{d} \varphi_{\mathrm{bal}}(\theta) / \mathrm{d} \theta=$ $a(b+c \cos \theta) /\left[(a \sin \theta)^{2}+(b \cos \theta+c)^{2}\right]-$ the derivative of $\varphi_{\text {bal }}(\theta)$ function by its argument; note that in the adopted conditions $\lambda(\theta)>0$, i.e. the function $\varphi_{\mathrm{bal}}(\theta)$ is increasing.

Using the approach for derivation of (7), it is possible to find the relationship which is inverse to $\varphi_{\text {bal }}(\theta)$ :

$$
\begin{gathered}
\theta_{\mathrm{bal}}=\sin ^{-1} \frac{c \tan \varphi}{\sqrt{a^{2}+(b \tan \varphi)^{2}}}+\tan ^{-1} \frac{b \tan \varphi}{a} \approx \\
\approx \sin ^{-1}\left(\varphi / \lambda_{0}\right) .
\end{gathered}
$$

To study the dynamics of $\theta$ angle change in accordance with the equation (7) when the controlled trailer moves forward $\left(V_{\text {car }}>0\right)$ and there are no external disturbance $\left(\Delta \dot{\psi}_{\text {tr }} \equiv 0\right)$. To do this, first we will consider the case when the vehicle steering wheels angle value satisfies the conditions: $\varphi \equiv$ const $<\varphi_{\text {bal }}(\theta)$. From relationship (7), it follows that it generates $\dot{\theta}<0$, i.e. decreasing of the angle $\theta$. As the $\varphi_{\text {bal }}(\theta)$ function is increasing, the function itself decreases. This will continue until there is the equation $\varphi=\varphi_{\text {bal }}\left(\theta_{\text {bal }}\right)$.

If we assume $\varphi \equiv$ const $>\varphi_{\text {bal }}(\theta)$ under the same conditions, it follows from (7) that $\dot{\theta}>0$, i.e. increase of the angle $\theta$. But this increase should be limited to the value $\left|\theta_{\text {bal }}\right| \leq \theta_{\max }<90^{\circ}$, otherwise $\varphi_{\text {bal }}(\theta)$ function will be decreasing and there will occur instability.

Therefore, sustainable movement requires fulfillment of the following condition: $\left|\theta_{\text {bal }}\right| \leq \theta_{\max }<90^{\circ}$. Hence, based on the $\varphi_{\mathrm{bal}}(\theta)$ function value according to (8) and on the increasing type of the function within the specified limits, we can generate the following requirement:

$$
\varphi_{\max }=\tan ^{-1} \frac{a \sin \theta_{\max }}{c+b \cos \theta_{\max }} \approx \lambda_{0} \sin \theta_{\max }
$$

This requirement can be interpreted as limiting the scope of deviation for the control steering wheels $\left(\varphi_{\max }\right)$ with given kinematic parameters; or with parameter $\varphi_{\max }$ given, to limit parameter $c$ which is the length of the trailer. If we consider that $\theta_{\max }=90^{\circ}$, in the first case: $\varphi_{\max }=\tan ^{-1}(a / c)$, in the second case: $c \leq a / \tan \varphi_{\max }$.

Further we assume that $\varphi_{\max }$ is set and represents a design limitation for steering wheels, and parameter $c$ meets the requirement (10), for example, for $\varphi_{\max }=30^{\circ}$ it is correct that $\mathrm{c} \leq 1.73 \mathrm{a}$. Then we can assume that during the forward movement and the absence of external disturbance, movement along the hitch angle is always stable. It should also be noted that $\theta_{\text {jack }}=\theta_{\text {max }}$, where the latter is defined by (9):

$$
\begin{aligned}
\theta_{\text {jack }}=\theta_{\max }= & \sin ^{-1} \frac{c \tan \varphi_{\max }}{\sqrt{a^{2}+\left(b \tan \varphi_{\max }\right)^{2}}}+ \\
& +\tan ^{-1} \frac{b \tan \varphi_{\max }}{a} \approx \sin ^{-1}\left(\frac{\varphi_{\max }}{\lambda_{0}}\right) .
\end{aligned}
$$

If the vehicle moves backwards $\left(\mathrm{V}_{\text {car }}<0\right)$ with the same conditions - taking such considerations into account, it follows that the system is definitely unstable; this explains all the problems with such movement control. In this case equation (7) will be the following: 


$$
\dot{\theta}=k(\theta) \cdot \sin \left[\varphi_{\mathrm{bal}}(\theta)-\varphi\right]-\Delta \dot{\psi}_{\mathrm{tr}} .
$$

We can find the trailer track curvature $(\kappa)$ out of the relations (4) and (5) obtained, in the absence of external disturbance:

$$
\kappa=\mathrm{R}_{\mathrm{tr}}^{-1}=\frac{\dot{\psi}_{\mathrm{tr}}}{\mathrm{V}_{\mathrm{tr}}}=\frac{a \sin \theta-b \cos \theta \tan \varphi}{c(a \cos \theta+b \sin \theta \tan \varphi)} .
$$

We can also find the trailer track curvature for steady movement (rotation) with $\varphi \equiv$ const $\neq 0$ value set and in the absence of external disturbance. From the foregoing, it follows that this movement corresponds to $\theta=\theta_{\text {bal }} \equiv$ const according to (9). In this case, we call the value of the trailer track curvature as a balancing value $\left(\kappa_{\mathrm{bal}}\right)$. It is easy to find out the following out of (8) and (13), by means of direct insertion:

$$
\kappa_{\mathrm{bal}}=\frac{\sin \theta_{\mathrm{bal}}}{b+c \cos \theta_{\mathrm{bal}}} \approx \eta \theta_{\mathrm{bal}}
$$

where $\eta \triangleq(b+c)^{-1}-$ is the proportionality coefficient; the approximate dependence of the equation obtained by linearization is about $\theta_{\mathrm{bal}}=0$.

Equations (1) - (14) with the accepted assumptions describe the kinematic and associated dynamic relations of the controlled trailer that are necessary for the control law synthesis for backup movement. Due to their definitions, the approximate equations in the relations according to (8), (9), (11) precisely describe the corresponding relationships for zero value angles. Upon angle modules increase, the tolerance increases, but the calculations show that the tolerance does not exceed $10-15 \%$ for typical practical problems with limitation $\varphi_{\max }=30^{\circ}$.

From formula (14), we can see that if the signal $\theta_{\text {bal }}$ is taken for a given value of hitch angle and tracked, it will approximately conform to the curvature control with a coefficient $\eta$ equal to the inverse distance between the rear axle and wheels axle of the trailer. Consequently, the use of hitch angle control instead of trailer track curvature control, unlike [7-9], can be interpreted as a variant of the automated backup movement. As to achievement of the required trailer track curvature, the driver can easily to correlate its value with a set hitch angle, based on experience of angular motion of the controlled trailer as it moves forward, and on the length of the object. The approximate nature of relation (14) is not critical, because the driver controls the movements by sight and operates the terms of more - less.

Such approach is used in the present work.

\section{CONTROL LAW}

Let us consider the control law options. At first, we assume that the parameters of the controlled object $\varphi_{\text {max }}, k_{\mathrm{st}}, a, b, c$ are known, and the set value for hitch angle (let us denote it as $\theta_{\text {set }}$ ) is generated by the driver. To eliminate the possibility of system jackknifing, this signal is limited on the basis of (11) by an obvious inequation:

$$
\left|\theta_{\text {set }}\right|<\sin ^{-1}\left(\frac{\varphi_{\max }}{\lambda_{0}}\right)-\Delta_{\theta} \leq \theta_{\text {jack }}
$$

where $\Delta_{\theta}$ is a small set positive number satisfying this inequation, to ensure no exceeding of $\theta_{\text {jack }}$.

We can offer a control law:

$$
\varphi_{\mathrm{st}}=k_{\mathrm{st}}^{-1}\left(\mathrm{k}_{\mathrm{ctrl}} \lambda_{0}\left(\sin \theta-\sin \theta_{\mathrm{set}}\right)+\varphi_{\mathrm{bal}}(\theta)\right)
$$

where $k_{\text {ctrl }}$ - is an assigned positive coefficient of the control law, which affects the speed of the transition process and reduction of external disturbances affect; we assume that this parameter stays within the limits:

$$
1 \leq k_{\mathrm{ctrl}}<\frac{\pi}{\left(2 \lambda_{0}\left|\sin \theta-\sin \theta_{\text {set }}\right|\right)} .
$$

This requirement follows from the subsequent reasoning.

Indeed, if we insert control law (16) into equation (12), we obtain the description of hitch angle dynamics in a closed control system during backup movement:

$$
\begin{aligned}
\dot{\theta} & =-k(\theta) \cdot \sin \left[k_{\mathrm{ctrl}} \lambda_{0}\left(\sin \theta-\sin \theta_{\mathrm{set}}\right)\right]-\Delta \dot{\psi}_{\mathrm{tr}} \approx \\
& -k(\theta) \cdot \sin \left[k_{\mathrm{ctrl}}\left(\varphi_{\mathrm{bal}}(\theta)-\varphi_{\mathrm{bal}}\left(\theta_{\mathrm{set}}\right)\right)\right]-\Delta \dot{\psi}_{\mathrm{tr}}
\end{aligned}
$$

where the approximate equation is given by the approximation to (8). An obvious requirement for control sustainability and proportionality, provided no disturbances are present, is that the sine argument module does not exceed the amount $\pi / 2$. This implies the upper limit (17).

Let us consider the exact relation (18), provided there is no external disturbance $\left(\Delta \dot{\psi}_{\mathrm{tr}} \equiv 0\right)$, with $\theta_{\text {set }}=$ const. It is not difficult to find out that $\theta \rightarrow \theta_{\text {set }}$ over time. This in turn results in $\varphi \rightarrow \varphi_{\text {bal }}\left(\theta_{\text {set }}\right) \equiv$ const according to (1) and (16). The control object stable behavior is obvious, in particular, sustainable rotation. Moreover, comparing the approximate part (18) with the forward movement dynamics equation (7), we can see that provided $k_{\text {ctrl }}=1$, the controlled trailer backup movement dynamics is approximately the same as for the forward movement with $\varphi=\varphi_{\text {bal }}\left(\theta_{\text {set }}\right)$. If $k_{\text {ctrl }}$ increases, speed of the step response increases too.

Thus, the control law (16) for controlled trailer backup movement under these conditions ensures stable system behavior, and in case of $k_{\mathrm{ctrl}}=1$ it ensures the same movement type as in the positive speed case.

If the trailer is affected by external disturbances $\Delta \dot{\psi}_{\mathrm{tr}} \neq 0$, in case of linear approximation, steady hitch angle value $\left(\theta_{\text {std }}\right)$ is expressed under a current mode (when $\dot{\theta} \cong 0$ ) in (18) as:

$$
\sin \theta_{\mathrm{std}} \approx \sin \theta_{\mathrm{set}}+\frac{\sin ^{-1}\left(\Delta \dot{\psi}_{\mathrm{tr}} / k(\theta)\right)}{\lambda_{0} k_{\mathrm{ctrl}}} \neq \sin \theta_{\mathrm{set}}
$$


Since $\theta_{\text {std }} \neq \theta_{\text {set }}$, other parameters of the movement will not match the expected ones. But equation (19) shows that the choice of $k_{\text {ctrl }}>1$ can weaken the influence of external disturbance.

Due to sight movement control, the control law can be simplified: instead of (16), given (8), we can write down:

$$
\varphi_{\mathrm{st}}=k_{\varphi}\left[k_{\mathrm{ctrl}}\left(\sin \theta-\sin \theta_{\mathrm{set}}\right)+\sin \theta\right],
$$

where $k_{\varphi} \triangleq \lambda_{0} / k_{\text {st }}$ is the proportionality coefficient.

It differs from the law (16) by approximate job functions only. $\varphi_{\text {bal }}(\theta)$. Let us consider what it gives. We rewrite (20) as

$$
\varphi_{\mathrm{st}}=k_{\mathrm{st}}^{-1}\left[k_{\mathrm{ctrl}} \lambda_{0}\left(\sin \theta-\sin \tilde{\theta}_{\mathrm{set}}\right)+\varphi_{\mathrm{bal}}(\theta)\right]
$$

where $\sin \tilde{\theta}_{\text {set }} \triangleq \sin \theta_{\text {set }}+\left(\varphi_{\text {bal }}(\theta)-\lambda_{0} \sin \theta\right) /\left(k_{\text {ctrl }} \lambda_{0}\right)$ is the new set hitch angle value, small offset due to the of destination $\varphi_{\text {bal }}(\theta)$ inaccuracy.

Here, similar reasoning suggests that $\theta \rightarrow \tilde{\theta}_{\text {set }} \equiv$ const $\neq$ $\theta_{\text {set, }}$ but if you increase parameter $\mathrm{k}_{\mathrm{ctrl}}$, the specified inaccuracy will decrease. Stability by the hitch angle is maintained.

\section{AUTO-SETUP MODE}

Implementation of the control law (20) requires information about the parameter $k_{\varphi}$. To determine it directly, the driver should perform a lot of measuring operations. To facilitate this process, it is proposed to organize the autodetection for this parameter, or a system auto-setup.

The auto-setup procedure is recommended to be organized during the test forward movement, in an arc, with no external disturbance, with the use of the parametric identification algorithm. To implement this algorithm, we use the hitch angle dynamic equation (6) for small angles $\varphi, \theta$ (e.g., not more than 10-15 degrees, provided the mobile device program selects such data itself) and provided $\Delta \dot{\psi}_{\mathrm{tr}} \equiv 0$. Then, given (1), this equation can be written as:

$$
\varphi_{\mathrm{st}} \approx \boldsymbol{\rho}^{\mathrm{t}} \mathbf{y}
$$

where $\boldsymbol{\rho}^{\mathrm{t}} \triangleq\left[k_{\varphi},\left(c k_{\varphi} / V_{\text {car }}\right)\right]$ is an unknown parameters vector, $\boldsymbol{y}^{\mathrm{t}} \triangleq[\theta, \dot{\theta}]$ is a regressors vector (in terms of regression analysis); superscript " $\mathrm{t}$ " denotes the transposition operation; signal $\dot{\theta}$ is determined by numerical differentiation $\theta$.

It is possible to use the classical least squares method as the most effective identification algorithm [14]:

$$
\widehat{\boldsymbol{\rho}}_{N}^{\mathrm{t}}=\left(\sum_{i=0}^{N} \varphi_{\mathrm{st}_{i}} \mathbf{y}_{i}^{\mathrm{t}}\right)\left(\sum_{i=0}^{N} \mathbf{y}_{i} \mathbf{y}_{i}^{\mathrm{t}}\right)^{-1}
$$

where the 'hat' over the symbol means its estimate; $i=1,2,3, \ldots, N$ are the numbers of discrete measurements and corresponding calculations with finite $N$, which, alternatively, may be automatically determined by means of an algorithmic analysis on the part of the evaluator of the fact of nondegeneracy achievement and a sufficient justification of the matrix which you need to use; temporary variables measurements discreteness is quite small in comparison with the system transition process.

The first element of a vector $\widehat{\boldsymbol{\rho}}_{N}$ is the desired estimate. If additional algorithmical data selection for case $\dot{\theta} \approx 0$ is organized for this movement, instead of (22), we can use:

$$
\hat{\mathrm{k}}_{\varphi} \approx \varphi_{\mathrm{st}} / \theta
$$

Based on the estimate $\hat{\mathrm{k}}_{\varphi}$, it is easy to define the limitation for setting of the required hitch angle from the non-jackknifing point of view. Based on (1), (11): $\hat{\theta}_{\text {jack }}=\varphi_{\text {st }_{\text {max }}} / \hat{\mathrm{k}}_{\varphi}$, and equation (15) will be rewritten for:

$$
\left|\theta_{\text {set }}\right|<\sin ^{-1}\left(\frac{\varphi_{\text {st }_{\text {max }}}}{\hat{\mathrm{k}}_{\varphi}}\right)-\Delta_{\theta} \leq \theta_{\text {jack }} .
$$

\section{MODEL STUDIES}

Let us assume the following parameters of the controlled object: $a=2.5 \mathrm{~m}, \quad b=0.5 \mathrm{~m}, \quad c=2 \mathrm{~m}, \quad \varphi_{\max }=30^{\circ}, k_{\mathrm{st}}=$ 0.055 . We also assume that together with the control signals production system the driver is described by an aperiodic link with unity gain, time constant of $0.2 \mathrm{~s}$ and permanent delay of $0.25 \mathrm{~s}$. Driver wheel deflection and hitch angle sensors have tolerance in the form of a centered white noise with standard deviation $0.3^{0}$.

Studies show that estimation $\hat{\mathrm{k}}_{\varphi}$ is determined by the proposed method (22) or (23) with the accuracy of at least 10\%. Fig.2 shows the control law (20) process with estimate $\hat{\mathrm{k}}_{\varphi}$ (error $\left.10 \%\right), \quad k_{\mathrm{ctrl}}=2$, when exposed by external disturbance. The simulation was performed in Matlab medium.

Studies confirm the above mentioned conclusions and demonstrate the control system target properties performance with tracking precision of a maximum preset hitch angle $3^{0}$ even in the presence of significant uncontrolled disturbance.

In addition to the presented results, the study of the mobile assistance system properties was conducted for a robotic model; this confirms the achievement of all the above mentioned system functional properties.

\section{CONTROL SYSTEM IMPLEMENTATION FEAURES}

Fig. 3 shows the structure of a driver assistance device.

The sensor of driver wheel deflection angle and the trailer hitch angle sensor are set before the start of trailer backup movement by the driver on the steering wheel and trailer hitch respectively using the quick mounting systems (magnets, hooks, adhesive tape, etc.) and are turned ON. The sensors transmit measurement signals to the mobile device by a wireless channel. 


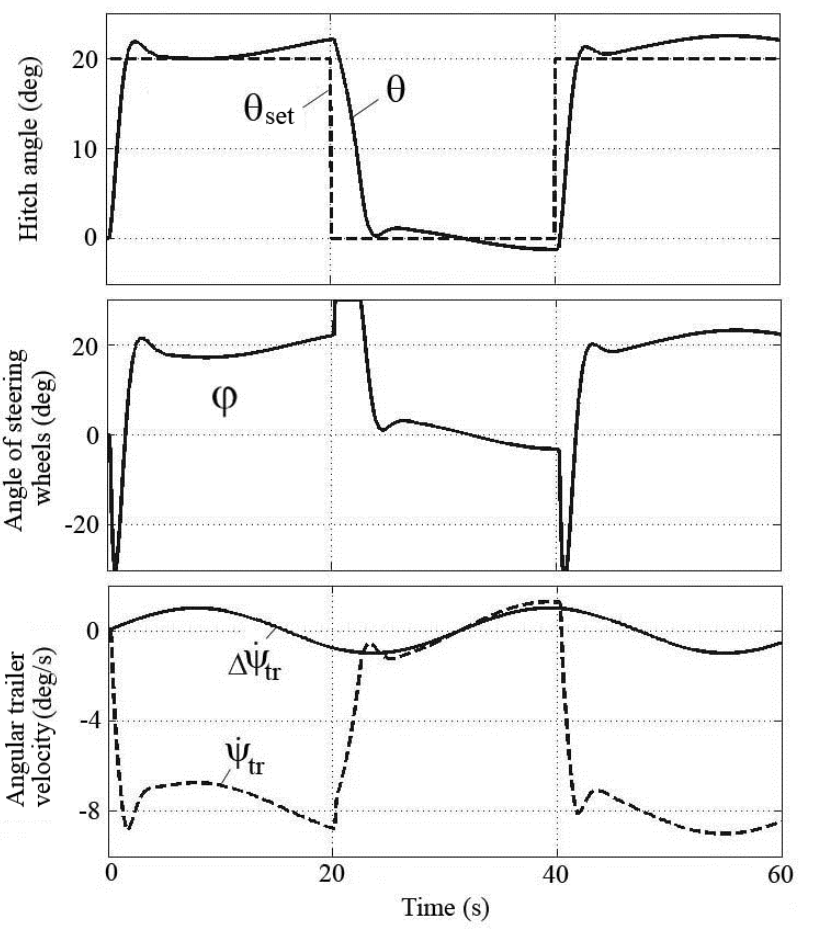

Fig. 2 Mobile assistance system control

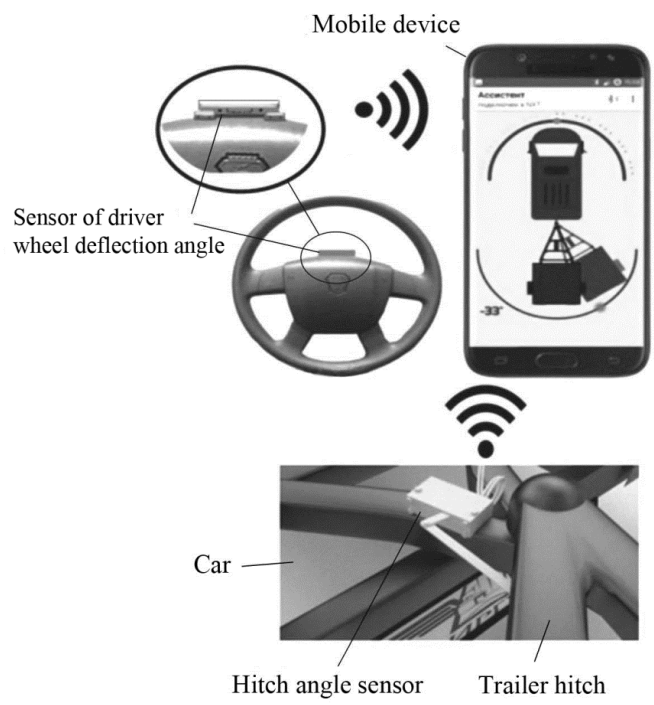

Fig. 3 Mobile Trailer Backup Assist System

The sensors may be constructed in various ways. The main criteria are: independence, wireless channel for communication with a mobile device, portability, replaceability, low cost.

One design variant of steering wheel rotation angle sensor is the implementation based on micromechanical sensor MPU6050 [15] which represents a triaxial angular rate sensor (gyro sensor) and a triaxial accelerometer. Since steering wheels rotation axis of modern cars is inclined from the vertical axis, we can create an independent and quite accurate steering angle sensor if we use a complementary filter on the basis of angular velocity and acceleration readings. In addition, the sensor has a microcontroller for data processing, a wireless transmission channel, and batteries.

One design variant of hitch angle sensor is an independent sensor with a microcontroller, wireless data transmission channel and batteries, with an optical encoder, rotary potentiometer, etc. as sensing elements. This sensing element receives vehicle and trailer longitudinal axis deflection angle hitch angle - through breakers or a flexible bridge (Fig.3).

The mobile device is mounted to a convenient for driver's observation place (dashboard, holder, etc.), and its specialized software is initialized. At first, if such information is present, control system parameters are introduced or adjusted to the mobile device: $k_{\mathrm{st}}, a, b, c$. If not, use the system auto-setting mode for test forward movement with small angles $\varphi, \theta$ according to the relationships (21-24). Next, with help of the mobile device, the following is entered: value $\varphi_{\text {st }}$ max coefficient $k_{\text {ctrl }}$ and the preset value of hitch angle $\left(\theta_{\text {set }}\right)$.

Mobile device limits the signals $\theta_{\text {set }}$ and $\mathrm{k}_{\mathrm{ctrl}}$ for dependencies (11), (15), (17), (24) and generates the required driver wheel deflection angle according to the relationship (20). In addition, by comparing the current driver wheel angle with the desired value, the mobile device gives the driver some commands (voice and/or dynamic image) on this angle adjustment. The driver controls the vehicle driving speed himself. Angle $\theta_{\text {set }}$ adjustment is also possible during the movement.

It should be noted that in comparison with (17) additional restriction of the value $\mathrm{k}_{\mathrm{ctrl}}$ is required. It is connected with a possibility of oscillatory unstability of the closed control system including the driver with individual characteristics on response to control signals. Therefore at emergence of fluctuations in system the driver has to reduce the specified coefficient.

Fig.4 shows an option of a human-machine interface implemented by means of a mobile device in the form of Assistant program.

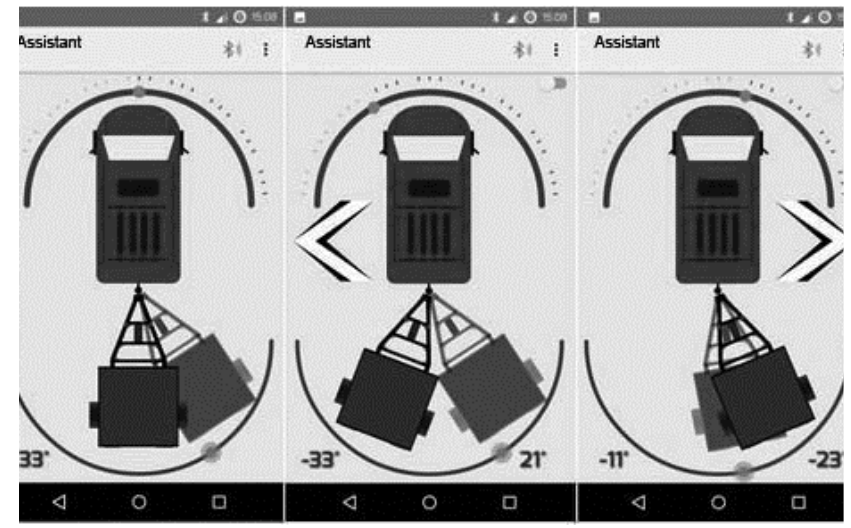

a)

b)

c)

Fig. 4 Human-machine interface for the "Assistant" program 
The interface is completed by means of several pages to configure and initialize the entire system, kinematic parameters tasks, $k_{\text {ctrl }}$ coefficient, auto-setting mode, setup of the required hitch angle and vehicle control by commands from the mobile device (Fig.4 shows some of them). Page a) demonstrates the procedure for entering the predetermined hitch angle by moving of trailer icon in the interactive monitor. Pages b) and c) show the procedure of generation of commands for the driver for the purpose of adjusting the driver wheel position and the current position of the trailer relative to the set one.

\section{CONCLUSION}

In general, it can be noted that the proposed version of the Mobile Trailer Backup Assist System is quite effective. It features such properties as low cost equipment (the mobile device is available practically for anybody), ease in installation and use.

\section{REFERENCES}

[1] M. Werling, P Reinish, M. Heidingsfeld, K.Gresser, "Reversing the general one-trailer system: asymptotic curvature stabilization and path tracking," IEEE Transactions on intelligent transportation systems,Vol.15, No.2, pp. 627-636, April 2014.

[2] M. Hafner, and T. Pilutti, "Control for Automated Trailer Backup," SAE Technical Paper 2017-01-0040, 2017.

[3] E.M. Lavoie, R.A. Trombley, C. Nave, J. Shutko, M.Y. Rupp, K.M. Mayer, Patent US 9248858 B2, 2016. Trailer backup assist system.
[4] S.G. Kong, B. Kosko, Adaptive Fuzzy Systems for Backing up a Truckand-Trailer, IEEE Transactions on neural networks, Vol. 3, No. 2, 1992, pp 211-223.

[5] J. Cheng, Y. Zhang, Z. Wan, "Curve path tracking control for tractortrailer mobile robot", Fuzzy Systems and Knowledge Discovery (FSKD) Eighth International Conference, July 2011, Vol. 1, pp. 502-506.

[6] Y. Bin, T. Shim, N. Feng, D. Zhou, "Path tracking control for backingup tractor-trailer system via model predictive control", Control and Decision Conference (CCDC) 2012 24th Chinese, May 2012, vol., no. pp. 198-203.

[7] R.A. Trombley, T.E. Pilutti, E.M. Lavoie, C. Nave, J. Shutko, R.Lanoue, B.G. Hochrein, N.Rolfes, Patent US 9037349 B2, 2015. Trailer identification system for trailer backup assist.

[8] E.M. Lavoie, D.S. Rhode, Patent US 9592851 B2, 2017. Control modes for a trailer backup assist system.

[9] Hafner, T.E. Pilutti, Patent US 9783230 B2, 2017. Trailer backup assist system with off-shoot correction.

[10] V. Josef, "Trailer parking assistant," Proceedings of the 16th International Conference on Mechatronics - Mechatronika 2014, Brno, 2014, pp. 677-682.

[11] E.M. Lavoie, C. Kyrtsos, Patent US 9335163 B2, 2016. Trailer length estimation in hitch angle applications.

[12] E.M. Lavoie, C. Kyrtsos, Patent US 9623904 B2, 2017. Trailer curvature control with adaptive trailer length estimation.

[13] L. Xu, E.H. Tseng, T.E. Pilutti, S.Y. Schondorf, D.D. Hrovat, J.P. Joyce, Patent US 9340228 B2, 2016. Trailer motion and parameter estimation system.

[14] L. Ljung, System Identification: Theory for the User. Second Edition. Prentice Hall PRT. Upper Saddle River, TO 07458.

[15] MPU-6000/MPU-6050 Product Specification. InvenSense, 2013, 54p. Website: www.invensense.com. 